\title{
La Sociedad Chilena de Anatomía. El Inicio
}

\author{
Chilean Society of Anatomy. The Beginning
}

Julio Cárdenas Valenzuela ${ }^{1,3}$ \& Alberto Rodríguez Torres ${ }^{2,3}$

CÁRDENAS, V. J. \& RODRÍGUEZ, T. A. La Sociedad Chilena de Anatomía. El Inicio. Int. J. Morphol., 36(3):1075-1078, 2018.

RESUMEN: Trayendo a la memoria hechos acaecidos hace un tiempo y con el fin de conservarlos para las futuras generaciones de morfólogos, es que son analizados los acontecimientos que condicionan el comienzo de la Sociedad Chilena de Anatomía, sus primeras directivas, publicaciones y reuniones, tanto nacionales como internacionales. Se realizó una búsqueda que incluyó relatos, revisión de estatutos, archivos digitales y revistas oficiales de la misma Sociedad, desde 1979 en que fue fundada hasta 1991, fecha en que coincidieron algunos cambios en las revistas, periodos de directorios y el fin de la presencia de los socios fundadores en los directorios y organización de los eventos futuros. Fueron revisados los primeros estatutos de la Sociedad, se hizo una descripción cronológica de los encuentros nacionales que derivaron posteriormente en congresos, evidenciando la evolución de las revistas de divulgación oficial de la misma y la instauración de reconocimientos hacia sus miembros más destacados.

PALABRAS CLAVE: Historia; Sociedad Chilena de Anatomía; Anatomía.

\section{Los preparativos: 1973-1978.}

Dos hechos están vinculados a la creación de una nueva Sociedad que agrupase nuevamente a los anatomistas chilenos, luego del intento fallido por mantenerse unidos con los anatomopatólogos (Rodríguez \& Cárdenas, 2017).

En el período posterior a la disolución de la antigua Sociedad de Anatomía Normal y Patológica (Espinoza \& Fuller, 2015), un pequeño grupo de anatomistas formados en la Universidad de Chile comenzó a reunirse en el domicilio del Prof. Dr. Abraham Wainstein. Allí concurrían habitualmente los profesores Dres. Humberto Guiraldes, Alberto Rodríguez y, ocasionalmente, el Dr. Hugo Hernández, radicado en Valdivia. En estas reuniones se divagaba acerca de la posible unión de los morfólogos de los diversos centros de Enseñanza Superior existentes a la fecha y que se distribuían en las Universidades de Chile, con sedes en Santiago, Valparaíso y Temuco, las Universidades Católicas en Santiago y Valparaíso, la Universidad de Concepción y la Universidad Austral en Valdivia. Aún no se tenía la separación de las Sedes Regionales de la Universidad de Chile (Antofagasta, Valparaíso, Talca y Temuco) que ocurriría en 1981. Largos años debieron pasar para que estas inquietudes se asentaran y motivaran a algunos anatomistas miembros de la Sociedad ya disuelta, así como a otros anatomistas en formación, para tener una entidad que organizara y promoviera la Morfología en Chile.
El otro hecho favorable para la creación de la nueva Sociedad fue que habiendo asistido por iniciativa personal el Prof. Dr. Alberto Rodríguez al V Congreso Panamericano de Anatomía efectuado en São Paulo en 1978, toma contacto con Profesores anatomistas Liberato DiDio y Eros Erhart, organizadores de dicho Congreso y, especialmente, con el Prof. Dr. José Carlos Prates, de la Escola Paulista de Medicina. El Prof. Dr. José Carlos Prates acepta recibir a anatomistas chilenos como profesores visitantes, alumnos de magíster y posteriormente de doctorandos en Ciencias, área de concentración en Anatomía. La Escola Paulista de Medicina fue un centro formador de morfólogos de primera categoría, con la inefable presencia del Profesor Prates.

También hubo esfuerzos individuales de formación anatómica de académicos en el extranjero (Francia- Italia) que no lograron difundir al resto de anatomistas, pero que ayudaron a elevar el nivel de la investigación nacional, como fue lo sucedido con el Prof. Hernández Parada (Rodríguez, 2014).

Al mismo tiempo, hubo participación en Congresos de la Sociedad Rioplatense de Anatomía lo que también amplía el horizonte e incentiva la investig,ación actualizada y la docencia, aumentando la masa crítica de morfólogos en el país, con una visión moderna de enseñanza e investigación aplicable en los nuevos campus docentes originados en 
el país (Actas de Directorio Sociedad Chilena de Anatomía Años 1979 a 1991, 2018).

Estas circunstancias se podrían considerar como los cimientos para explicar el nacimiento de la futura Sociedad Chilena de Anatomía, y sería la propuesta para la resolución de problemáticas internas recibiendo influencias foráneas.

Fundación de la Sociedad (1979). En vista de la organización, metódicas de investigación y avances de la docencia observados en el extranjero, el 13 de octubre de 1978 se reunieron los morfólogos macro y microscopistas como respuesta a la convocatoria del Profesor Alberto Rodríguez, en una Jornada Anatómica en el Campus Occidente de la Universidad de Chile, Santiago, a la cual asistieron 104 personas. Esta Jornadase se extendió todo el día, al término de la cual se fundó la Sociedad Chilena de Anatomía culminando con una reunión social de camaradería en un restaurant céntrico de Santiago. En tal Jornada se escucharon diversas opiniones y propuestas de los asistentes sobre cómo realizar la docencia e investigación de la Anatomía, para así generar consensos. Se acordó efectuar una reunión al inicio del año siguiente (1979) para originar la fundación de una nueva sociedad que acogiese a todos los morfólogos chilenos (Rodríguez, 2016).

Primer Encuentro de Anatomía. Se realizó en abril de 1979, en el anfiteatro de Anatomía del Instituto de Anatomía de la Escuela de Medicina de la Universidad de Chile (Cárdenas, 2006), lugar donde se dio el puntapié inicial a la Sociedad Chilena de Anatomía y se nominó al Prof. Dr. Abraham Wainstein como Presidente. Se acordó proseguir con la realización de Encuentros Nacionales de Anatomía, los que posteriormente (1990) pasaron a ser denominados Congresos Chilenos de Anatomía. La personería jurídica de la Sociedad se obtuvo el 12 de diciembre de 1979.

Ese mismo año, se materializó el comité organizador de la Sociedad Chilena de Anatomía Patológica, la que se constituyó como tal al año siguiente, el 14 de mayo de 1980 (Sociedad Chilena de Anatomía Patológica, 2018).

La nueva Sociedad Chilena de Anatomía constituyó su Directorio de la siguiente forma:

Presidente Prof. Dr. Abraham Wainstein Sabat, Universidad de Chile.

Vice-presidente Prof. Dr. Alberto Rodríguez Torres, Universidad de Chile.

Secretario Prof. Dr. Patricio Quezada Pacheco, Universidad de Chile.

Tesorero Prof. Dr. Robinson González Fernández, Universidad de Chile.

Directores: Profs. Drs. Evaristo Cancino Águila, Universidad de Chile. Hernán Ceppi, Universidad de Chile; Jorge Safián, Universidad de Chile, Humberto Guiraldes, Pontificia Universidad Católica de Chile. Hugo Hernández Parada, Universidad Austral; Atilio Almagiá Flores, Universidad Católica de Valparaíso; Humberto Cerisola, Universidad Católica de Valparaíso. (Actas de Directorio Sociedad Chilena de Anatomía Años 1979 a 1991).

\section{Consolidación de la Sociedad.}

a) Encuentros Nacionales de Anatomistas, Congresos internacionales. Se realizaron los Encuentros Nacionales de Anatomistas en las siguientes Sedes y de responsabilidad de los socios de la respectiva Universidad:

I Encuentro Nacional de Anatomía 1979, Santiago de Chile, Universidad de Chile, Sede Norte.

II Encuentro Nacional de Anatomía 1980, Santiago de Chile, Universidad Católica de Chile.

III Encuentro Nacional de Anatomía 1981, Valdivia, Universidad Austral.

IV Encuentro Nacional de Anatomía 1982, Temuco, Universidad de la Frontera. (Asisten Profs. Drs. José Carlos Prates, de Brasil y Alfredo Ruiz Liard, Uruguay, entre otros.)

V Encuentro Nacional de Anatomía 1983, Concepción, Universidad de Concepción.

VI Encuentro Nacional de Anatomía 1984, Punta del Este, Uruguay (en conjunto con el VII Congreso de la Asociación Panamericana de Anatomía) (Losardo, 2009).

VII Encuentro Nacional de Anatomía 1985, Mendoza, Argentina, en conjunto con el Congreso de la Asociación Rioplatense de Anatomía.

VIII Encuentro Nacional de Anatomía 1986, Valparaíso, Universidad Católica de Valparaíso.

IX Encuentro Nacional de Anatomía 1987, Santiago, Universidad de Chile, en conjunto con el VIII Congreso Panamericano de Anatomía.

X Encuentro Nacional de Anatomía.1988, Valdivia, Universidad Austral.

XI Encuentro Nacional de Anatomía1989, Concepción, Universidad de Concepción.

XII Congreso Chileno de Anatomía 1990, Trujillo Perú, en conjunto con el IX Congreso Panamericano de Anatomía.

XIII Congreso Chileno de Anatomía 1991, Temuco, Universidad de La Frontera.

Paulatinamente, se incorporaron académicos e investigadores procedentes de antiguas sedes regionales de la Universidad de Chile como Antofagasta y Talca, y de la primera universidad privada con Facultad de Medicina: la Universidad de los Andes, de Santiago (1991). Cabe destacar que, producto de ello, desde sus inicios hasta nuestros días, nunca se ha dejado de realizar anualmente una actividad equivalente a un encuentro de carácter nacional.

De estos Encuentros nacionales y Congresos interna- 
cionales, tanto de los efectuados en el país como de los realizados en el extranjero, se produjeron múltiples anécdotas relacionadas con los precarios medios de transporte y alojamiento a los que debieron echar mano parte de los participantes chilenos, dada la modesta colaboración que se obtenía por parte de las autoridades que dirigían las diferentes universidades.

Especial connotación tuvo el VIII Congreso Panamericano de Anatomía realizado en Santigo, entre el 28 y 31 de octubre de 1987, conjuntamente con el IX Encuentro Nacional de Anatomía. Ejecutado bajo la presidencia del Prof. Dr. Alberto Rodríguez Torres, congregó a más de trescientos anatomistas provenientes de diez países americanos incluyendo a Estados Unidos de Norteamérica.

Ingreso a la Federación Internacional de Asociaciones de Anatomía. Desde agosto de 1980, en Ciudad de México y gracias a la participación personal del entonces Secretario de la Federación Internacional de Asociaciones de Anatomía (IFAA) Profesor Dr. Liberato Di Dio, la Sociedad Chilena de Anatomía representada por el Prof. Dr. Hugo Hernández, pasó a ser miembro activo de esta Federación mundial.

Directorios: Con el tiempo se logró realizar varias reuniones de trabajo y de directorio en el curso de cada año, y una reunión anual obligatoria, en la que el Presidente de la Sociedad daba cuenta de lo realizado en ese período. El mandato del Presidente de la Sociedad era de dos años, pudiendo ser reelecto por una vez. La costumbre era elegir al Vicepresidente como Presidente en el ciclo posterior. Las citaciones para esta elección debían ser efectuadas anualmente, a través de dos publicaciones en un periódico de circulación general y en un período determinado.

\section{Presidentes y Vicepresidentes de la Sociedad Chilena de Anatomía entre los años 1979 y 1992.}

1979-1980. Presidente. Abraham Wainstein Sabat. Vicepresidente Alberto Rodríguez Torres.

1981-1983. Presidente Alberto Rodríguez Torres. Vicepresidente Humberto Guiraldes del Canto.

1984-1985. Presidente Humberto Guiraldes del Canto. Vicepresidente Jorge Safián Pino.

1986-1987. Presidente Humberto Guiraldes del Canto. Vicepresidente Hugo Hernández Parada

1988-1989 Presidente Hugo Hernández Parada. Vicepresidente Hernán Ceppi Kahler.

1990-1991. Presidente Fernando Matamala Vargas. Vicepresidente Alberto Rodríguez Torres.

Medios de divulgación:Los Archivos Chilenos de Morfología, (1933-1950) creación del Profesor dr. Gustavo Jirón en 1933, Diciembre, (archivos chilenos, 1942), tuvieron una edición discontinuada correspondiendo el último Archivo al Nº 8 que se publicó en 1950 (Rodríguez \& Cárdenas).

Los Anales de Anatomía Normal (1983-1990) vinieron a llenar el vacío existente y a la necesidad de dar a conocer los resúmenes de los trabajos presentados en los Encuentros de Anatomía originados desde 1979. En 1983, durante la presidencia del Dr. Rodríguez se dio a conocer el $\mathrm{N}^{\circ} 1$ de la publicación que llevó el nombre de Anales.

«Los proyectos son ambiciosos y se han trazado etapas con metas progresivas: a los Resúmenes de los Encuentros, ya realizados, le siguen los Anales que incluirán los Trabajos presentados; posteriormente y junto con mantener los Anales, seguirá la Revista que incluirá un material de alta selección y que deberá cumplir con las normas internacionales que rigen este tipo de publicaciones para ser consideradas como fuente bibliográfica obligada».

Los «Anales de Anatomía Normal» consistía en una publicación anual, editada en Las Palmeras 299, al interior de la Quinta Normal, dirección que correspondía a la Sede de Medicina Occidente de la Universidad de Chile. Como norma inicial, no se recibían trabajos que no correspondiesen a los presentados en los Encuentros y Congresos para su publicación. Es así como se editaron estos Anales durante 1983 a 1989. En 1987, la publicación incluyó 46 trabajos (Rodríguez, 2005). Al año siguiente se publicaron 53 trabajos distribuidos en 8 áreas:

Histología: 15 artículos; Embriología: 4 artículos; Anatomía Comparada: 9 artículos; Morfología Experimental: 8 artículos; Técnica Microscópica: 4 artículos; Antropología Física: 4 artículos; Educación en Morfología 7 artículos; Anatomía y Arte: 2 artículos.

Los Editores responsables de la publicación de los Anales de Anatomía Normal en el periodo 1983 y 1988 fueron los Profs. Drs. Alberto Rodríguez Torres y Hernán Ceppi Kahler junto a Robinson González y de la Revista Chilena de Anatomía, de 1989 en adelante, Mariano del Sol C. (del Sol, 1999). Además, participaron en los Comités Editorial y de Redacción los Profs. Drs. Humberto Guiraldes del Canto de la Pontificia Universidad Católica de Chile y Hugo Hernández Parada de la Universidad Austral de Chile.

En 1990, los Anales Chilenos de Anatomía cambian su nombre y pasan a nominarse Revista Chilena de Anatomía (1990-2002) teniendo por Editor al Prof. Dr. Mariano del Sol, quien había obtenido los grados académicos de Magister y Doctor en Ciencias Morfológicas (Anatomía) en la Escola Paulista de Medicina bajo la tutoría del Prof. Dr. José Carlos Prates. 
Estatuto. Desde la creación de la Sociedad Chilena de Anatomía, existe el Estatuto, aprobado en abril de 1979 e inscrito ante notario en noviembre de 1979, contenplando su modificación acorde con las necesidades que se presentaran.

Están contemplados en su articulado todas las características propias de la Sociedad como entidad científica, sus fines, características de sus miembros, cargos, elección de las autoridades, las posibilidades de modificación de los artículos acorde a las necesidades que se estimen adecuadas y su disolución. Hasta 1991, el estatuto fue modificado en dos oportunidades: $1^{\text {a }}$ modificación en noviembre de 1979 y $2^{a}$ modificación en mayo de 1980.

\section{Reconocimientos de la Sociedad}

Maestros de la Anatomía. Desde su fundación en 1979 hasta el año 1991, diversos directorios de la Sociedad han determinado reconocer en vida o en forma póstuma, la trayectoria de algunos profesores nacionales o extranjeros que han destacado por la impronta dejada en relación a servicios prestados a la Anatomía nacional, por el desempeño excepcional en cargos de la propia Sociedad o desde el extranjero, para lo cual se instauró el premio de Maestro de la Anatomía. Así, se han distinguido en este periodo con este premio, máximo reconocimiento de sus pares, a los profesores que han dejado su impronta en la Morfología nacional a través de sus cargos societarios y de carrera docente, anatomistas como: Abraham Wainstein y Adolfo Escobar Pacheco reconocidos en 1983 y 1984, respectivamente (Revista Chilena de Anatomía, 1998). Gustavo Jirón Latapiat, 1987. Enrique Solervicens 1987 y Carlos Henckel de Concepción, propuesto pero no nombrado.

En un comunicado realizado por Comunicaciones de la Pontificia Universidad Católica de Chile en relación al Prof. Dr. Adolfo Escobar Pacheco, en reunión de Anatomistas previo al VI Congreso Panamericano de Anatomía efectuado en Uruguay (Punta del Este) octubre 1984, se señalaba "Cabe destacar que esta es la segunda vez que la Sociedad Chilena de Anatomía distingue como socio honorario con el Título de Maestro de Anatomía a uno de sus miembros siendo el primero de ellos el Dr. Abraham Weinstein. La distinción se entrega a aquellos profesionales nacionales cuya labor docente y de investigación haya tenido relevancia o influencia en el progreso de la Anatomía en el país."

CÁRdENAS, V. J. \& RODRÍGUEZ, T. A. Chilean society of anatomy. The beginning. Int. J. Morphol., 36(3) :1075-1078, 2018.

SUMMARY: With the aim of bringing to mind events that occurred some time ago, and in order to preserve them for future generations of morphologists, the authors make an analysis of the facts that conditioned the beginnings of the Chilean Society of Anatomy such as, the society's first board of directors, publications and national and international meetings. A search was carried out that included anecdotes, reviews of statutes, digital archives and official society journals, from 1979 when it was established, until 1991 the year during which some changes were introduced in the journals, the duration periods for the board of directors and the termination of the founding partners' participation on the board and organization of future events. It was possible to review the society's initial statutes, make a chronological description of the national meetings and subsequent congresses, and of the society's official journals and establishment of recognitions towards its most outstanding members.

KEY WORDS: History; Sociedad Chilena de Anatomía; Anatomy.

\section{REFERENCIAS BIBLIOGRÁFICAS}

Actas de Directorio Sociedad Chilena de Anatomía Años 1979 a 1991. Santiago de Chile, Sociedad Chilena de Anatomía, 2017.

Cárdenas, V. J. El Instituto de Anatomía de la Universidad de Chile. An. Chil. Hist. Med., 16(1):33-42, 2006.

del Sol, C. M. Editorial. Rev. Chil. Anat., 17(2):133-4, 1999.

Espinoza, A. \& Fuller, A. Anatomía Patológica, 132 Años de Historia, Huella y Presencia. Santiago de Chile, Facultad de Medicina Universidad de Chile, 2015.

Homenaje póstumo al Profesor Doctor Adolfo Escobar Pacheco. Maestro de Anatomía. 1905-1998. Rev. Chil. Anat., 16(2):279-80, 1998.

Losardo, R. J. Pan-American Association of Anatomy: Historic review and eminent rules. Int. J. Morphol., 27(4):1345-52, 2009.

Rodríguez, T. A, Entrevista. Santiago de Chile, Instituto de Anatomía, Agosto 2014.

Rodríguez, T. A. \& Cárdenas, V. J. The origins of the Society of Normal and Pathological Anatomy of Chile. Int. J. Morphol., 35(2):751-5, 2017.

Rodríguez, T. A. Discurso de agradecimiento del Profesor Dr. Alberto Rodríguez Torres, al nombramiento como Maestro de la Anatomía de Chile. Int. J. Morphol., 23(1):43-4, 2005.

Rodríguez, T. A. Entrevista. Santiago de Chile, Instituto de Anatomía, Mayo 2016.

Sociedad Chilena de Anatomía Patológica. Sitio Web. Santiago de Chile, Sociedad Chilena de Anatomía Patológica, 2018. Disponible en: http:/ /schap.cl/institucion/historia

Dirección para correspondencia:

Dr. Julio Cárdenas

Anatomía y Biología del Desarrollo

Facultad de Medicina

Universidad de Chile

Santiago

CHILE

Email: jcardena@med.uchile.cl

Recibido : 22-11-2017

Aceptado: 14-04-2018 